\title{
Les formes graphiques de l'insécurité L'exemple de l'accent circonflexe
}

\author{
Marie-Anne Paveau \\ Université Paris 13, Villetaneuse \\ EA 452 Cenel
}

\begin{abstract}
«Enlever les accents circonflexes, c'est comme si dans une vieille demeure Renaissance vous refaites un linteau qui s'écroule en béton coulé. C'est insensé ! Ça dénature! » (rapporté par Valérie Feschet, "Les délices de l'orthographe.... »)

«Dans l'Express d'aujourd'hui, il manque un chapeau à "hôpital" ॥ (modérateur du forum de http://www.ile-maurice.com, aout 2002)
\end{abstract}

\section{Introduction}

L'hypercorrection est une attitude langagière motivée par un sentiment d'insécurité linguistique, concept introduit par William Labov dans Sociolinguistique en $1976^{1}$ : un scripteur fortement conscient de la norme (comme l'est, selon Pierre Bourdieu 1979, celui qui appartient à la « petite bourgeoisie ") et craignant de faire des fautes, ajoute sans nécessité fonctionnelle des marques graphiques, en général celles ressenties comme relevant de la norme de prestige (l'accent circonflexe, le h étymo-graphique, certains $s$ après consonne muette). Ces marques contiennent selon Berrendonner 1998 un fort " potentiel de valorisation ».

Je me propose dans cet article de rendre compte de cette attitude graphique des scripteurs du français sous la pression de la norme académique omniprésente dans l'ensemble des secteurs de la société concernés par l'écrit en France. J'ai choisi l'angle apparemment anecdotique de l'accent circonflexe : après une synthèse rapide sur les enjeux linguistiques et sociaux du fameux chapeau, je tenterai d'expliquer que cette attitude complexe croise plusieurs dimensions (psychique, cognitive, sociale et esthétique), qui constituent des obstacles ou des écrans à une pratique raisonnable de la graphie du français, ainsi qu'à son évolution. Je présenterai un corpus d'hypercorrections concernant l'accent circonflexe, à propos duquel je montrerai que ces phénomènes sont toujours pluri-dépendants : de la matérialité graphique de l'énoncé en cours, du contexte linguistique immédiat, de l'histoire de la langue et de l'environnement psycho-socionormatif. Ce corpus rassemble des énoncés appartenant à divers contextes scripturaux. Mon regard n'est pas centré ici sur l'enseignement-apprentissage ; mon travail vise plutôt à proposer, à partir d'un phénomène socio-scriptural commun aux scripteurs du français, des pistes pour une intégration des attitudes sociolinguistiques à l'enseignement de l'orthographe du français ${ }^{2}$.

\section{Les zones d'insécurité de la graphie du français}

Le couplet sur l'accent circonflexe est un passage obligé de tous les manuels, vade mecum, guides, essais et discours sur la qualité de la langue (pour une synthèse sur ces discours, voir Paveau, Rosier 2008). La plupart du temps, le discours est militant, et vise à la conservation de

\footnotetext{
${ }^{1}$ Sur l'origine et la caractérisation de la notion, voir Berrendonner 1998.

${ }^{2}$ Le Français aujourd'hui applique les rectifications orthographiques de 1990, qui concernent comme on le sait les accents circonflexes sur les $i$ et $u$. C'est le cas dans cet article, dans les énoncés que j'assume énonciativement ; mais ce n'est pas le cas dans les citations et les exemples donnés en corpus, où j'analyse des emplois de circonflexes superflus, qui se font dans un contexte cognitif d'où les rectifications, qui n'ont jamais été diffusées en France, sont cognitivement absentes.
} 
l'accent menacé par les réformes, les nouvelles technologies de l'écriture et l'inéluctable dégradation de toutes choses. Un exemple entre mille, dans un ouvrage collectif publié au moment de la publication des Rectifications de 1990 :

Il y a cet incomparable accent circonflexe, si menacé. A lui seul, il justifierait bien des polémiques et des refus. Tout le monde aura remarqué avec quelle insistance il a été évoqué dans tous les débats récents qui ont eu lieu autour de l'orthographe. II est clair que c'est un point sensible. A juste titre. II suffit de relire L'Huître de Francis Ponge pour voir combien toute la poésie, et au-delà peut-être toute la langue, peut se trouver suspendue à l'accent circonflexe. Alors épargnons-le, choyons-le. II est de ces petits signes qui nous aident encore à respirer, à nous amuser et à retrouver notre enfance, c'est-à-dire la continuité de notre vie (Collectif [Raymond Jean] 1990, p. 53).

II se trouve que l'accent circonflexe, et plus généralement les accents tout court, constituent l'une des zones d'instabilité et d'insécurité du français (avec, entre autres, les doubles consonnes et les homophones) $)^{3}$. Les spécialistes de l'écrit et de la graphie le savent bien, et savent aussi que ce sont souvent ces zones de turbulence qui sont la cause des fautes, et non une prétendue insuffisance cognitive ou culturelle des scripteurs (même si celle-ci est repérable par ailleurs). Nina Catach a bien décrit la fonction désormais réduite de ce petit signe suscrit :

Aujourd'hui, cet accent a pratiquement perdu tout rapport avec une indication de longueur, sauf dans les paires spécifiques comme pâte/patte, côte/cote, etc., où certains locuteurs continuent, suivant en cela la norme, à sentir une opposition. En dehors du français de certaines régions, Belgique, Suisse, Canada, etc., l'accent circonflexe ne conserve aujourd'hui, pour la plupart des Français, que la seule fonction d'opposition phonémique, hôte/hotte, tâche/tache, jeûne/jeune, etc., le reste étant senti comme simple rappel étymologique ou historique de lettres ou d'hiatus disparus (Catach (dir.) 1995, p. 1129).

Ce « rappel étymologique » constitue l'argument le plus souvent mis en avant pour défendre le maintien du circonflexe. Cela revient à maintenir un signe pour en signaler un autre, qui n'existe plus. Dans un autre contexte, on appellerait cela un mémorial, ou une stèle, voire une " couronne mortuaire » (l'expression est de Ferdinand Brunot).

Maurice Tournier propose de lui attribuer une fonction " graphologique », cependant réduite à 18 cas :

Phonologiquement sans objet ou remplaçable par l'accent grave dans $99,99 \%$ des cas où il serait utile, l'accent circonflexe correspondrait-il davantage à une fonction " graphologique », en entendant par là l'apport de sens produit par une simple différence graphique ? Dix-huit quasi-homographes dans toute la langue française répondent seuls à l'appel: tète/tête, gène/gêne, pèche/pêche, cocher/côcher, colon/côlon, cote/côte, coté/côté, rot/rôt, roder/rôder, faite/faîte, parait/paraît, boite/boîte, croit/croît, gite/gîte, gitons/gîtons, mimes/mîmes, primes/prîmes, rimes/rîmes (Tournier 1991, p. 103).

Pour résumer, l'accent circonflexe cumule les étrangetés : il est d'usage compliqué, comme en témoignent les kilomètres de discours sur son usage (et cela tant à l'écrit qu'à l'écran, sur les traditionnels supports papier comme sur les modernes forums, sites et blogs) ; il est inutile sauf dans les 18 quasi-homographes que nous signale Maurice Tournier; il favorise les fautes d'orthographe et plonge les scripteurs en état d'insécurité linguistique. Citons encore Maurice Tournier sur ce point :

Les distinctions grapho-sémantiques n'ont dans tous les autres cas jamais besoin du supplément du circonflexe ; car là encore, pour un son identique, ou bien cette accentuation est superflue, d'autres graphes s'avérant distinctifs (près/prêt, grasse/grace, haute/hote/ote, cou/coud/coup/out, il/ile, mettre/mètre/maitre, plaie/plait), ou bien l'accent grave suffit aux distinctions (foret/forèt, belle et

\footnotetext{
${ }^{3}$ Sur la question des zones d'instabilité graphique, voir Jaffré, Fayol 1997. Dans les rectifications de 1990, la suppression du circonflexe sur les voyelles $i$ et $u$ est motivée par la régularisation de manière à réduire ces zones d'instabilité : dans la mesure où il n'est pas discriminant (c'est-à-dire qu'il ne sert pas à distinguer des homophones comme dans sur et sûr, ou du et $d \hat{u}$, ni à indiquer une marque de conjugaison comme la forme fût du subjonctif par rapport à la forme fut du passé simple), il peut disparaître sans que la langue n'y perde rien ; en revanche les usagers y gagnent en maîtrise du code.
} 
bèle, freine/frène, bas/bàt, moud, mou, mout, moue et moùt, mur/mùr). La circonflexion ne fait qu'ajouter à la complexité orthographique sans ajouter rien à la signification des mots (Tournier 1991, p. 103).

Alors à quoi sert-il ? Et surtout, pourquoi cette "relation passionnelle avec l'accent circonflexe », comme l'écrit l'auteure du blog la-minute-encyclopédique. pensée du jour, écrits et images ${ }^{4}$, dans le titre de l'un de ses billets?

Bernard Cerquiglini a fourni en 1995, quelques années après ce qu'il appelle « la cruelle guerre civile qui divisa la France " (allusion aux débats qui ont accompagné le texte de 1990), une savante et exhaustive explication de cette passion française dans son livre L'accent du souvenir. II y raconte toute l'histoire du circonflexe qui contient elle-même (presque...) toute l'histoire de la graphie du français.

II explique par exemple que le fameux $s$ dont la trace historique est rappelée par le circonflexe (bastir, Benoist, beste, conqueste, coste, creistre, ostel, Pasques, Pentecoste, etc.), est lui-même une sorte d'ombre graphique : "Pour les phonéticiens en effet, le son [s] ainsi noté, et que l'on entendait par exemple dans le latin bestia et le très ancien français beste, a disparu de la prononciation autour de $1066 »$ (p. 11). On comprend donc que le militant contemporain du circonflexe se bat pour le rappel d'un son disparu... près de dix siècles auparavant. Alors pourquoi en maintenir si fermement la mémoire ? Bernard Cerquiglini explique, en se basant sur d'anciens traités d'orthographe, que l's est maintenu pour des raisons spécifiquement orthographiques (rapport de l'écrit à lui-même ou à la morphologie) et non phonographiques (rapport de l'écrit à l'oral), servant essentiellement à distinguer le passé simple (fit, lut) de l'imparfait du subjonctif (fist, lust); l's est également conservé pour des raisons esthétiques, pour la " belle écriture ». On comprend donc mieux cet attachement passionnel au circonflexe à l'aube du 21e siècle, ressortissant à la transmission d'une préoccupation esthétique fixée sur la consonne $s$, et surtout à la naissance même de la logique orthographique ainsi décrite par Bernard Cerquiglini :

L's, placé devant le $t$ (avec lequel, dans l'écriture médiévale, il forme ligature), est beau ; il est bon de l'employer, sans autre raison; [...] l's implosif est une butte-témoin, la dernière résistance à l'érosion phonique, une pièce maintenue de l'ossature du mot. Utiliser un tel signe, de pure graphie, c'est noter une appartenance; l'employer par la seule grâce de l'effet esthétique, c'est figurer l'appartenance générale de la langue, faire allégeance, la valoriser. [...] Employé par la graphie médiévale, transmis à l'orthographe renaissante, l's préconsonantique ne transcrit pas un son. II est un lieu de mémoire et d'hommage, l'inscription d'une esthétique et d'une culture. Plus qu'une graphie, c'est un graphisme. En cela, il est au fondement de l'orthographe française, tout comme l'accent circonflexe, qui lui succèdera (Cerquiglini 1995, p. 35 et 36).

On saisit donc que l's implosif et son héritier le circonflexe organisent partiellement la naissance de l'orthographe comme pratique de réglementation de l'écrit, pratique qui comporte une dimension esthétique. II y a là une explication forte du maintien du circonflexe et du fréquent discours conservateur sur les accents, qui passe souvent par l'argument étymologiste. Ce n'est pas pour autant une justification: Bernard Cerquiglini considère que « l'étymologisation, tendance ancienne et constante de l'orthographe française, est [...] une tentative désespérée, car nécessairement intermittente et disparate, de réduire par l'écrit la béance qu'a creusée l'évolution phonique propre au français " (p. 95). Mais en linguiste documenté, historien de la langue et observateur des usages et des représentations, il montre que le petit signe est le lieu d'un enjeu important, qui est la nature même de la graphie française. Le circonflexe est décoratif, c'est un fait, et les conservateurs ont tort, selon Bernard Cerquiglini, de ne pas le dire plus clairement. Mais cet ornement figure selon lui « l'ambigüité de l'orthographe française, prise depuis toujours entre l'écrit et l'oral, les lettres et les sons, la mémoire et l'oubli » (p. 161). Et il allie de manière paradoxale mais bien réelle son inutilité linguistique à sa légitimité culturelle : "Le circonflexe, figure double au destin paradoxal, est l'icône tutélaire de cette orthographe équivoque, qui arbore et vénère un signe que plus rien ne justifie, mais que tout légitime » (p. 161).

\footnotetext{
${ }^{4}$ http://laminute.canalblog.com/archives/2006/07/05/2069010.html
} 


\section{Quelques exemples de circonflexes superflus}

Entrons dans l'analyse des attitudes des scripteurs qui fixent inconsciemment leur inquiétude graphique sur ce petit chevron, en procédant à ce qu'A. Berrendonner appelle des " substitutions ornementales " (Berrendonner 1998). Soit le corpus suivant constitué d'ajouts de circonflexes inutiles sur le $i$ (toutes les graphies sont conservées y compris les autres fautes; l'orthographe rectifiée n'est pas appliquée dans ce corpus) :

[1] Publicité pour la maison Hédiard recueillie dans un magazine en décembre 2005

Faîtes partager des émotions

Hédiard, leader de l'épicerie fine, c'est 5 boutiques et plus de 150 points de vente en France. Notre enseigne crée l'émotion en faisant de chacun de ses produits un cadeau à part entière. Pour la période magique de Noël, nous renforçons nos équipes, de décembre à janvier [...].

\section{[2] Courriel}

Date : Wed, 21 Jan 2009 11:08:59 +0100

À : (Liste des destinataires supprimée )

Chères et chers collègues,

Si vous faîtes circuler l'annonce des sommaires des dernier et prochain numéros de notre revue, merci de bien vouloir utiliser cette dernière version du document, quelques coquilles ayant dû être corrigées.

Bien cordialement.

[auteur : professeur des universités en science politique]

[3] Page d'accueil de site internet (les deux phrases citées sont enchainées)

http://www.rue-des-sims.com/

Toutes les créations sont faites par re-des-sims

Faîtes comme chez vous!

\section{[4] Courriel}

Date : Wed, 07 May 2008 16:15:27 +0200

À : Marie-Anne Paveau <marie-anne.paveau@libertysurf.fr>

Bonjour,

Effectivement, nous envoyons une fiche de demande de service de presse aux revues susceptibles d'être intéressées mais n'hésitez pas à en intégrer dans votre liste (nous croiserons les deux fichiers, le vôtre et le nôtre). Je pense que 30 cartes, ce sera un peu juste... Dîtes-le moi si tel est le cas.

[auteur : éditeur]

\section{[5] Courriel}

Date : Wed, 23 May 2008 13:23:45 +0200

À : Marie-Anne Paveau <marie-anne.paveau@libertysurf.fr>

Bonjour madame Paveau,

Après une longue absence, je suis de retour à Paris.

Je ne reste pas longtemps ici, je rentre chez moi la fin de ce mois...donc bientôt.

Je voulais vous voir pour discuter un peu avec vous et vous demander conseil.

Dîtes-moi quand est-ce que je pourrais venir.

[auteur : doctorant]

[6] Titre d'article sur site internet

http://www.centpapiers.com

1 juillet 2010

Le Panda: Mr Sarkozy, dîtes nous tout !

\section{[7] Transcription chanson sur site personnel}

http://bmarcore.perso.neuf.fr

Dîtes moi ma Mère - Willemetz-Yvain

Chantée par Maurice Chevalier, 1928

Dîtes moi ma mère

Dîtes moi ma mère

Pourquoi les chiens dans la rue

Se montent dessus?

Dîtes moi ma mère 
Dîtes moi ma mère

Pourquoi qu'on leur jette sur l'dos

Des grands seaux d'eau ?

Dîtes moi c'est curieux pourquoi les éléphants

Ont une queue derrière et une autre devant?

Dîtes moi ma mère

Laquelle qui leur sert

Pour montrer qu'ils sont content ?

[8] Titre article presse quotidienne en ligne

http://www.leparisien.fr

Rubrique VOCABULAIRE, 30.03.2010

Ne dîtes plus «buzz» mais «ramdam»

\section{[9] Courriel - liste de discussion}

Date : Wed, 10 Dec 2008 09:28:33 +0100

À : multitudes-infos@samizdat.net

Objet : Re: [multitudes-infos] Manifestation devant le consulat de Grèceà Paris

bonjour,

diable, je ne suis pas certain que ce forum soit le lieu propice à un

tel déballage de vos pulsions intîmes.

Soit maintenant la liste des mots français qui contiennent un $i$ accentué avec le circonflexe (liste issue de Wikipedia, article " Accent circonflexe en français ", élaborée à partir des dernières éditions des dictionnaires courants ; je la considère comme fiable mais indicative) :

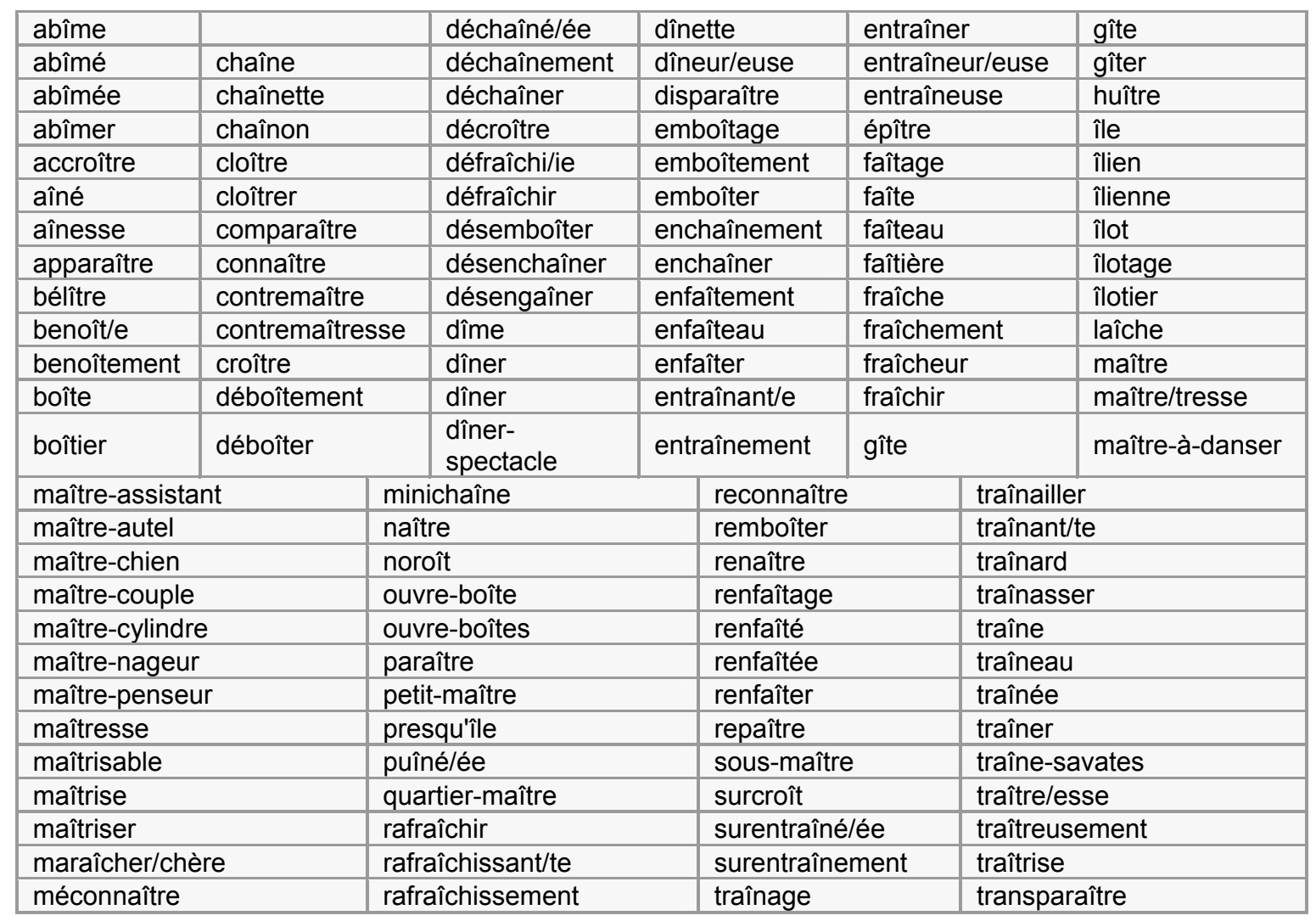

On peut faire les remarques suivantes pour rendre compte des hypercorrections circonflexes sur le $i$ :

- dans la majeure partie des cas, il existe un phénomène d'analogie, qui régit très souvent la " faute d'orthographe » : faîtes s'explique évidemment par la quantité importante de circonflexes en contexte graphique -aît-, bien apparente dans la liste, et éventuellement, mais la forme est moins courante, au mot « faîte »; dîtes croise la série morphologique dîn- et vraisemblablement la forme de passé simple dîtes; les choses sont sans doute plus complexes pour intîmes puisque 
seul abîme présente un support d'analogie ; le mot est sans doute perçu comme relevant d'un registre élevé.

- les exemples donnés impliquent tous une surveillance langagière particulière: les énoncés publiés sont en général des titres, particulièrement visibles, et sur lesquels se focalise donc une attention graphique redoublée (la publication et la position saillante du titre) ; les courriels sont soit collectifs, donc soumis à une lecture semi-publique (ceux des enseignants), soit adressés au sein d'une relation hiérarchique (doctorant-encadrant par exemple).

- enfin la matérialité de l'outil d'écriture joue un rôle : dans tous les cas, il s'agit du clavier, soit en contexte d'écriture privée comme le courriel, ou en contexte de rédaction journalistique ou assimilée comme pour la presse et les sites internet. Je n'ai pas intégré d'exemples manuscrits, comme je l'avais prévu avant d'écrire cet article, pour une raison simple: je n'ai trouvé aucun exemple d'hypercorrection graphique avec le circonflexe dans l'ensemble des copies d'étudiants que j'ai corrigées pendant l'année 2009-2010. Camille Martinez, travaillant sur les livres d'or avec graphies manuscrites spontanées, donne une explication à cela en constatant que les accents y sont souvent omis :

On peut avancer l'hypothèse que le système accentuel du français n'est pas utilisé dans toute sa latitude parce que sa mise en œuvre diminue la vitesse d'écriture, et parce que son absence n'empêche pas la lecture (sauf quelques cas d'ambigüité). En un mot, il n'est pas pertinent (Martinez 2007, p. 37).

Sur des graphies avec outil informatique, et en contexte surveillé, on constate l'inverse, ce qui implique que ces signes, et c'est tout particulièrement le cas du circonflexe, n'ont pas grand chose à voir avec le système de la langue mais tout à voir avec des facteurs psychiques (sécurité/insécurité) et sociaux (prestige/stigmatisation), auquel s'ajoute un facteur technique : lorsque l'on rédige à partir d'un clavier, le circonflexe est obtenu par une double manipulation (deux touches), alors que les accents grave et aigu sont frappés directement; le marquage du circonflexe est donc bien le produit d'un soin particulier apporté à la rédaction et d'une forte considération pour la norme.

Plus rares sont les circonflexes superflus sur $u$, a et $o$. Pour chacun des exemples suivants, donnés à titre indicatif, on pourra proposer le même type d'explication que précédemment c'est-àdire l'analogie, l'intériorisation de la marque de prestige, et la contribution de l'outil d'écriture :

[10] Légende de photo dans la presse

Le plexiglas de Kauffmann a chû (Midi libre, 05.08.2006 ; légende d'une photo d'œuvre d'art ayant été cassée au musée du centre Georges Pompidou en aout 2006)

[11] Extrait d'une biographie d'un DRH dans l'industrie

$\mathrm{X}, \mathrm{DRH}$ de l'année 2006

Par BERTAND LE BALC'H

$15 / 10 / 2007$

«SI en 1974, quand je me suis lancé dans la vie active, quelqu'un m'avait dit que je serais un jour DRH, je ne l'aurais pas crû », reconnaît X, 55 ans.

\section{[12] Programme de colloque}

11h15-12h00 Henning Schmidgen (Max-Planck-Institut für Wissenschaftsgeschichte): "La pensée cognitiviste dans la psychologie de Wundt."

$12 \mathrm{~h} 00-12 \mathrm{~h} 15$ : discussion

12h15-14H00 : déjeûner

\section{[13] Courriel}

Date : Sun, 8 Mar 2009 09:14:03 +0100

À : "Marie-Anne Paveau" < marie-anne.paveau@libertysurf.fr> [et autres]

Concernant le règlement intérieur, j'ai pour ma part quelques suggestions de modif et d'ajoûts notamment [auteur : professeur des universités en sciences économiques]

[14] Courriel [graphie entièrement conservée]

Date : Fri, 13 Mar 2009 20:58:11 +0200

À : "Marie-Anne Paveau" <marie-anne.paveau@libertysurf.fr> 
Merci bein de me donner ces petits renseignents qui me permettront d'être à l'heure et ne pas râter le séminaire.

Cordialement. [auteur : professeur des universités turc]

[15] Dépliant présentant les loisirs du port d'Aigues-Mortes

" prix de location du bâteau salon nautique 2003 »

\section{[16] Légende d'image télévisée}

http://langue-fr.net [site de défense du bon français]

Vu chez Pernaut-13h [NDÉ : journal télévisé de la première chaîne nationale française], une jeune fille préparant l'option « basque » au bac ; son nom en sous-titre, suivi de future bâchelière.

\section{[17] Extrait de presse}

http://langue-fr.net [site de défense du bon français]

Je relève aujourd'hui dans le portrait de Michel Drucker en dernière page de Libération : "Sa côte remonte comme celle du Formica chez l'antiquaire branché ».

\section{Le circonflexe : un bon sujet de conversation}

Le puriste ou sentinelle de la langue chasse le circonflexe hypercorrectif, comme nous l'indiquent les deux derniers exemples; on trouve en effet, je le rappelais plus haut, de très nombreux discours et commentaires sur l'accent circonflexe, comme cet amusant billet des correcteurs du Monde sur leur blog Langue sauce piquante, qui commente involontairement l'exemple [11] cidessus :

\section{Les carottes ont crû}

Quoi de plus simple ? Croire, cru ; croître, crû. Pour le participe passé, laissez-vous guider par l'accent. Et pourtant, au bal des cru-crû, quelle salade ! La plupart du temps, crû perd son « flexe »*, sans doute jugé trop encombrant.

II faut reconnaître que quelques dérivés de croître qui ont perdu leur chapeau ne facilitent pas les choses : le cru (le terroir), de son cru (de son invention), ou la crue (la montée des eaux d'un cours d'eau). Mais croyez-nous, si les carottes ont poussé, c'est qu'elles ont crû.

*'L'accent circonflexe, chez les correcteurs.

(http://correcteurs.blog.lemonde.fr/2005/09/29/2005_09_post_16/)

Mais le scripteur ordinaire manifeste également un évident souci circonflexif. Sur le site consacré au téléphone BlackBerry, appelé familièrement BB (www.blackberry-france.com), on trouve au moins deux discussions sur les moyens techniques de produire un circonflexe à l'écran. (http://www.blackberry-france.com/forum/l-atelier/accent-circonflexe-t961.html). La première (7 messages) commence par le post d'un utilisateur qui ne parvient pas à poser son chapeau sur sa lettre, et elle dérive rapidement vers le thème de la qualité de la langue. La seconde se clôt sur une hiérarchie des outils d'écriture électronique, le BB étant considéré comme supérieur au téléphone ordinaire, parce qu'il possède " un véritable clavier ». Je donne ici un aperçu de ces discussions en ligne (août 2008 : les titres sont les pseudos des forumers) :

\section{Première discussion}

\section{- Beogles}

Sujet du message: accent circonflexe

J'ai un blackberry curve. J'ai trouvé l'accent circonflexe dans les symboles, mais je n'arrive pas à le placer au-dessus de la lettre. L'accent se met avant, ou après, mais pas au-dessus.

Quel est le truc? merci.

- joshua

j'en profite pour exposer un petit problème.

Quand j'écris un sms (codé en 7 bits), je ne peux pas utiliser les circonflexes et autres figures.

Je peux le faire quand je passe en UCS2 mais mes textos ne passent pas tout le temps... Est-ce normal ?

- neod 
le système UCS2 est prévu au départ pour l'écriture cyrillique qui prend beaucoup plus d'octet en codage 7 bits

donc pour eux moins de lettre disponible par sms malgré cela il n'on que 70 caractère par sms contre nos 160

alala que de mauvais cote se cyrillique

- joshua

C'est donc normal qu'en 7 bits, je ne puisse pas écrire en parfait Français ?

C'est tout de même très con de leur part pour le coup...

\section{Seconde discussion}

- neoworld

A noter aussi que le langage sms est simple et pas français ... si vous voyez la où je veux en venir!

- LTDB

Tout à fait ! Alors nous, avec les BB, qui avons un véritable clavier, pourquoi devrions-nous nous priver d'écrire dans la langue de Molière ? A l'exception près de l'accent circonflexe et du "c" cédille Amicalement.

Ces échanges sont intéressants en particulier parce qu'ils vont à l'encontre du discours ambiant sur la dégradation de la langue dans l'écriture électronique : nos utilisateurs de BlackBerry, qui ont un peu le sentiment d'avoir entre les mains la Rolls du téléphone portable, font preuve d'une attitude linguistique tournée vers le prestige: ils se montrent très attachés à une graphie traditionnelle et respectueuse des normes, graphie qui les distingue apparemment du tout-venant des producteurs de sms (voir ici même l'article de Cédrick Fairon et Jean-René Klein). Nous ne disposons pas encore de corpus constitué de messages écrits sur BlackBerry (ou un autre type de téléphone intelligent) pour y relever d'éventuels circonflexes d'hypercorrection, mais il y a fort à parier que les résultats d'une telle étude réserveraient quelques surprises.

\section{Conclusion}

Dans ce travail, j'ai voulu insister sur les points suivants, qu'il me semble important de prendre en compte dans l'enseignement-apprentissage de l'écrit, en particulier sur les plan graphique et orthographique :

- Les scripteurs outillés par clavier manifestent une surveillance langagière qui semble supérieure aux scripteurs manuscrits spontanés; contrairement aux idées reçues, c'est plutôt l'écriture électronique que l'écriture manuscrite qui conserve la norme académique, en tout cas dans les contextes analysés ici. La rapidité et la simplicité de la frappe laisse sans doute davantage de temps aux scripteurs au clavier de se poser les questions qui mènent à l'hypercorrection.

- L'école faisant encore une très large place à l'écriture manuscrite, il est intéressant de mettre en rapport les régimes d'écriture avec des régimes d'usage de l'accentuation (extensible aux autres signes graphiques du français, comme la ponctuation). Imposer une norme orthographique unique alors que les scripteurs sont désormais tous, qu'ils soient en formation ou insérés professionnellement, dans des plurirégimes graphiques, est devenu contre-productif. L'orthographe des scripteurs serait plus sécure s'ils étaient autorisés à pratiquer la plurigraphie.

\section{Bibliographie}

- BERRENDONNER A. (1998), "Normes d'excellence et hypercorrections », Cahiers de Linguistique française 20, 87-101.

- BOURDIEU P. (1979), La distinction. Critique sociale du jugement, Paris, Les Éditions de Minuit.

- CATACH N. (dir.) (1995), Dictionnaire historique de l'orthographe française, Paris, Larousse.

- Collectif, 1990, Contre la réforme de l'orthographe. Va-t-on se laisser mordre la langue ?, collection Temps-choc, Marseille, éditions Autres temps. 
- Conseil supérieur de la langue française (1990), "Les rectifications de l'orthographe », Journal officiel de la République française, Édition des documents administratifs, arrêté du 6 décembre 1990.

- CERQUIGLINI B. (1995), L'accent du souvenir, Paris, Minuit.

- FESCHET V. (2002 [1998]), «Les délices de l'orthographe ou la passion des français pour la dictée de Pivot », dans BROMBERGER C. et al., Passions ordinaires. Du match de football au concours de dictée, Paris, Bayard, p. 189-217.

- JAFFRÉ J.-P., FAYOL M. (1997), L'orthographe, des systèmes aux usages, Paris, Flammarion.

- LABOV W., 1976 (1972), Sociolinguistique, Minuit.

- MARTINEZ C. (2007), « Recherches dans un corpus de français écrit naturel : le règne des agents de l'orthographe ", Cahiers AFLS, Volume 13 Issue 1, p. 33-46, revue en ligne: http://www.afls.net/cahiers/cover13.1.php?lang=fr

- PAVEAU M.-A., ROSIER L. (2008), La langue française. Passions et polémiques, Paris, Vuibert.

- PAVEAU M.-A (2008), «Le parler des classes dominantes, objet linguistiquement incorrect? Dialectologie perceptive et linguistique populaire », Études de linguistique appliquée 150, p. 137-156.

- PAVEAU M.-A (2009), « Quand Marie-Chantal dit merde : sentiment linguistique et normes perceptives dans la haute société ", Sentiment linguistique et discours spontanés sur le lexique, Metz, coll. « Recherches linguistiques », p. 41-63.

- TOURNIER M. (1991), «À quoi sert l'accent circonflexe ? », Mots 28, "Orthographe et société », p. 101-107. 